\title{
Intersection curve of two parametric surfaces in Euclidean $n$-space
}

\author{
Mustafa Düldül And B. Merih ÖZÇETin
}

\begin{abstract}
The aim of this paper is to study the differential geometric properties of the intersection curve of two parametric surfaces in Euclidean $n$-space. For this aim, we first present the $m$ th order derivative formula of a curve lying on a parametric surface. Then, we obtain curvatures and Frenet vectors of the transversal intersection curve of two parametric surfaces in Euclidean $n$-space. We also provide computer code produced in MATLAB to simplify determining the coefficients relative to Frenet frame of higher order derivatives of a curve.
\end{abstract}

\section{Introduction}

Recently, the surface-surface intersection problem has attracted much attention due to its importance in computer aided geometric design. This attention is due to the fact that determining the intersection curve, i.e. its parametric representation, of two surfaces is not easy in most cases. However, even if the intersection curve cannot be represented parametrically, it has been shown in recent studies that the differential geometric properties of the intersection curve can be still computed. If the tangent planes of two intersecting surfaces overlap at an intersection point, the intersection is called a tangential intersection, otherwise it is called a transversal intersection in Euclidean 3 -space $\mathbb{E}^{3}$. The majority of the recent studies focus on transversal intersection rather than tangential intersection. The reason for this is the easy computation (vector product of normal vectors of two surfaces) of the tangential direction in transversal intersection.

Differential geometry of intersection curves of two parametric surfaces in Euclidean 3-space has been studied by some authors using different methods

Received April 5, 2021.

2020 Mathematics Subject Classification. 53A05.

Key words and phrases. Surface-surface intersection, transversal intersection, Frenet vectors, curvatures.

https://doi.org/10.12697/ACUTM.2021.25.17

Corresponding author: B. Merih Özçetin 
$[10,21]$. These studies have been extended to $\mathbb{E}^{4}$ for the intersection of three parametric hypersurfaces $[6,9,10,14]$ and to $\mathbb{E}^{5}$ for the intersection of four parametric hypersurfaces [15]. Since surfaces can also be defined by their implicit equations, differential geometry of the intersection curve of two implicit surfaces has been studied in $\mathbb{E}^{3}$ by $[2,12,20,21]$ and of three implicit hypersurfaces has been studied in $\mathbb{E}^{4}$ by $[3,4,7,14,19]$. There also exist some studies for the intersection curves of different type surfaces in $\mathbb{E}^{3}$ $[10,18,21]$ and in $\mathbb{E}^{4}[1,8,10,14]$. On the other hand, by using the wedge product of two vectors in $(n+1)$-dimensions, Goldman [12] derived a closed formula for the first curvature of the transversal intersection of $n$-implicit hypersurfaces in $(n+1)$-dimensions. Aléssio [5] derived the second and the third curvatures of intersection curves of $(n-1)$-implicit hypersurfaces in $\mathbb{E}^{n}$ by generalizing the method of Goldman. Recently, the intersection curve of $(n-1)$ transversally intersecting hypersurfaces has been studied in $\mathbb{E}^{n}$ by [17].

If we take into account all of these recent studies, it is seen that all intersection problems have been considered for the intersection of $(n-1)$ hypersurfaces in $\mathbb{E}^{n}$. However, to the best of our knowledge, differential geometry of the intersection curve of two surfaces has not been studied in higher dimensions. Since the normal space of a surface is $(n-2)$-dimensional and the vector product is defined for $(n-1)$-vectors in $n$-space, computing the tangential direction even for the transversal intersection of two surfaces in $\mathbb{E}^{n}$ is not as evident as in $\mathbb{E}^{3}$.

The purpose of this paper is to consider the intersection problem of two parametric surfaces in $\mathbb{E}^{n}, n \geq 4$, and study the differential geometry of their intersection curve. First we obtain the $m$ th order derivative formula for a curve lying on a parametric surface. We also generate orthogonal bases for the normal spaces of the intersecting surfaces. By using the $m$ th order derivative formula and orthogonal bases of normal spaces of the intersecting surfaces, we present a method which enables us to compute all curvatures and Frenet vectors of the intersection curve of two parametric surfaces in $\mathbb{E}^{n}$. We also provide a computer code produced in MATLAB to simplify determining the coefficients relative to the Frenet frame of higher order derivatives of a curve.

This paper is organized as follows. Section 2 includes the definition of the vector product of $(n-1)$-vectors in $\mathbb{R}^{n}$, Frenet formulas and derivatives up to order 4 of a curve lying on a surface in $\mathbb{E}^{n}$. We present the $m$ th order derivative formula of a curve lying on a parametric surface in Section 3. Section 4 involves a method which enables us to compute all curvatures and Frenet vectors of the intersection curve of two parametric surfaces in $\mathbb{E}^{n}$. We provide two examples as an application of the method in Section 5. 


\section{Preliminaries}

Definition 1. Let $\left\{\mathbf{e}_{1}, \mathbf{e}_{2}, \ldots, \mathbf{e}_{n}\right\}$ denote the standard basis in $\mathbb{R}^{n}$. The vector product of the vectors $\mathbf{a}_{1}=\sum_{j=1}^{n} a_{1 j} \mathbf{e}_{j}, \mathbf{a}_{2}=\sum_{j=1}^{n} a_{2 j} \mathbf{e}_{j}, \ldots, \mathbf{a}_{n-1}=$ $\sum_{j=1}^{n} a_{n-1, j} \mathbf{e}_{j}$ is defined by (see [16])

$$
\mathbf{H}=\mathbf{a}_{1} \times \mathbf{a}_{2} \times \cdots \times \mathbf{a}_{n-1}=\left|\begin{array}{cccc}
\mathbf{e}_{1} & \mathbf{e}_{2} & \cdots & \mathbf{e}_{n} \\
a_{11} & a_{12} & \cdots & a_{1 n} \\
a_{21} & a_{22} & \cdots & a_{2 n} \\
\vdots & \vdots & & \vdots \\
a_{n-1,1} & a_{n-1,2} & \cdots & a_{n-1, n}
\end{array}\right| .
$$

The product $\mathbf{H}$ in $\mathbb{R}^{n}$ is a vector perpendicular simultaneously to all the $\mathbf{a}_{i}(1 \leq i \leq n-1)$ and its norm is given by the formula (see [16])

$$
\|\mathbf{H}\|=\left\|\mathbf{a}_{1}\right\| \cdot\left\|\mathbf{a}_{2}\right\| \ldots\left\|\mathbf{a}_{n-1}\right\| \cdot K,
$$

where

$$
K=\left|\begin{array}{cccc}
1 & \cos \alpha_{12} & \cdots & \cos \alpha_{1, n-1} \\
\cos \alpha_{21} & 1 & \cdots & \cos \alpha_{2, n-1} \\
\vdots & \vdots & & \vdots \\
\cos \alpha_{n-1,1} & \cos \alpha_{n-1,2} & \cdots & 1
\end{array}\right|^{1 / 2}
$$

with $\cos \alpha_{i j}=\frac{\left\langle\mathbf{a}_{i}, \mathbf{a}_{j}\right\rangle}{\left\|\mathbf{a}_{i}\right\| \cdot\left\|\mathbf{a}_{j}\right\|}$.

Let $\mathcal{S} \subset \mathbb{E}^{n}$ be a regular surface given by $X\left(u_{1}, u_{2}\right)$ and $\alpha$ be a unit-speed curve with the arc-length $s$ lying on $\mathcal{S}$. If we denote the Frenet frame of $\alpha$ by $\left\{\mathbf{V}_{1}(s), \mathbf{V}_{2}(s), \ldots, \mathbf{V}_{n}(s)\right\}$, then the Frenet formulas are given by (see [11])

$$
\begin{aligned}
& \mathbf{V}_{1}^{\prime}(s)=k_{1}(s) \mathbf{V}_{2}(s), \\
& \mathbf{V}_{i}^{\prime}(s)=-k_{i-1}(s) \mathbf{V}_{i-1}(s)+k_{i}(s) \mathbf{V}_{i+1}(s), \quad 2 \leq i \leq n-1, \\
& \mathbf{V}_{n}^{\prime}(s)=-k_{n-1}(s) \mathbf{V}_{n-1}(s),
\end{aligned}
$$

where $k_{1}(s), k_{2}(s), \ldots, k_{n-1}(s)$ are the curvatures of $\alpha(s)$. Using the Frenet formulas, we can write the derivatives of the curve $\alpha$ as

$$
\begin{aligned}
& \alpha^{\prime}=\mathbf{V}_{1}, \\
& \alpha^{\prime \prime}=k_{1} \mathbf{V}_{2}, \\
& \alpha^{\prime \prime \prime}=-k_{1}^{2} \mathbf{V}_{1}+k_{1}^{\prime} \mathbf{V}_{2}+k_{1} k_{2} \mathbf{V}_{3}, \\
& \alpha^{(4)}=-3 k_{1} k_{1}^{\prime} \mathbf{V}_{1}+\left(-k_{1}^{3}+k_{1}^{\prime \prime}-k_{1} k_{2}^{2}\right) \mathbf{V}_{2}+\left(2 k_{1}^{\prime} k_{2}+k_{1} k_{2}^{\prime}\right) \mathbf{V}_{3}+k_{1} k_{2} k_{3} \mathbf{V}_{4}, \\
& \vdots \\
& \alpha^{(n)}=\{\ldots\} \mathbf{V}_{1}+\{\ldots\} \mathbf{V}_{2}+\ldots+\{\ldots\} \mathbf{V}_{n-1}+k_{1} k_{2} k_{3} \ldots k_{n-1} \mathbf{V}_{n} .
\end{aligned}
$$


In addition, since the curve $\alpha(s)$ lies on the surface $\mathcal{S}$, we can write $\alpha(s)=$ $X\left(u_{1}(s), u_{2}(s)\right)$. Then we have

$$
\begin{gathered}
\alpha^{\prime}(s)=\sum_{i_{1}=1}^{2} X_{i_{1}} u_{i_{1}}^{\prime}, \\
\alpha^{\prime \prime}(s)=\sum_{i_{1}=1}^{2} X_{i_{1}} u_{i_{1}}^{\prime \prime}+\sum_{i_{1}, i_{2}=1}^{2} X_{i_{1} i_{2}} u_{i_{1}}^{\prime} u_{i_{2}}^{\prime}, \\
\alpha^{\prime \prime \prime}(s)=\sum_{i_{1}=1}^{2} X_{i_{1}} u_{i_{1}}^{\prime \prime \prime}+3 \sum_{i_{1}, i_{2}=1}^{2} X_{i_{1} i_{2}} u_{i_{1}}^{\prime \prime} u_{i_{2}}^{\prime}+\sum_{i_{1}, i_{2}, i_{3}=1}^{2} X_{i_{1} i_{2} i_{3}} u_{i_{1}}^{\prime} u_{i_{2}}^{\prime} u_{i_{3}}^{\prime}, \\
\alpha^{(4)}(s)=\sum_{i_{1}=1}^{2} X_{i_{1}} u_{i_{1}}^{(4)}+4 \sum_{i_{1}, i_{2}=1}^{2} X_{i_{1} i_{2}} u_{i_{1}}^{\prime \prime \prime} u_{i_{2}}^{\prime}+3 \sum_{i_{1}, i_{2}=1}^{2} X_{i_{1} i_{2}} u_{i_{1}}^{\prime \prime} u_{i_{2}}^{\prime \prime} \\
+6 \sum_{i_{1}, i_{2}, i_{3}=1}^{2} X_{i_{1} i_{2} i_{3}} u_{i_{1}}^{\prime \prime} u_{i_{2}}^{\prime} u_{i_{3}}^{\prime}+\sum_{i_{1}, i_{2}, i_{3}, i_{4}=1}^{2} X_{i_{1} i_{2} i_{3} i_{4}} u_{i_{1}}^{\prime} u_{i_{2}}^{\prime} u_{i_{3}}^{\prime} u_{i_{4}}^{\prime},
\end{gathered}
$$

where $X_{i}=\frac{\partial X}{\partial u_{i}}, \quad X_{i j}=\frac{\partial^{2} X}{\partial u_{j} \partial u_{i}}, \ldots$

\section{Higher order derivatives of a curve lying on a parametric surface}

We will need higher order derivatives of a curve lying on a surface. For that reason, we start by presenting the following theorem which enables to calculate higher order derivatives of a surface curve in $\mathbb{E}^{n}$.

Theorem 1. Let $\mathcal{S} \subset \mathbb{E}^{n}, n \geq 4$, be a regular surface given by its parametric equation $X\left(u_{1}, u_{2}\right)$ and $\alpha$ be a unit-speed curve with arc-length s lying on $\mathcal{S}$ given by $\alpha(s)=X\left(u_{1}(s), u_{2}(s)\right)$. Then, the mth order derivative of $\alpha$ is obtained by

$$
\begin{aligned}
\alpha^{(m)}=\mathcal{C}_{1} & \sum_{i_{1}=1}^{2} \frac{1}{k_{i_{1}}} X_{i_{1}} u_{i_{1}}^{(m)}+\sum_{r_{1}=1}^{\left\lfloor\frac{m}{2}\right\rfloor}\left(\mathcal{C}_{2} \sum_{i_{1}, i_{2}=1}^{2} \frac{1}{k_{i_{1} i_{2} r_{1}}} X_{i_{1} i_{2}} u_{i_{1}}^{\left(m-r_{1}\right)} u_{i_{2}}^{\left(r_{1}\right)}\right) \\
& +\sum_{r_{1}, r_{2}=1}^{\left\lfloor\frac{m}{2}\right\rfloor}\left(\mathcal{C}_{3} \sum_{i_{1}, i_{2}, i_{3}=1}^{2} \frac{1}{k_{i_{1} i_{2} r_{1} i_{3} r_{2}}} X_{i_{1} i_{2} i_{3}} u_{i_{1}}^{\left(m-r_{1}-1\right)} u_{i_{2}}^{\left(r_{2}\right)} u_{i_{3}}^{\left(r_{1}-r_{2}+1\right)}\right) \\
& +\sum_{r_{1}, r_{2}, r_{3}=1}^{\left\lfloor\frac{m}{2}\right\rfloor}\left(\mathcal{C}_{4} \sum_{i_{1}, i_{2}, i_{3}, i_{4}=1}^{2} \frac{1}{k_{i_{1} i_{i_{1}} i_{3} i_{2} i_{2} i_{r_{3}}}} X_{i_{1} i_{2} i_{3} i_{4}} u_{i_{1}}^{\left(m-r_{1}-2\right)} u_{i_{2}}^{\left(r_{2}\right)}\right. \\
& \left.\times u_{i_{3}}^{\left(r_{3}\right)} u_{i_{4}}^{\left(r_{1}-r_{2}-r_{3}+2\right)}\right)
\end{aligned}
$$




$$
\begin{array}{r}
+\sum_{r_{1}, r_{2}, \ldots, r_{m-2}=1}^{\left\lfloor\frac{m}{2}\right\rfloor}\left(\mathcal{C}_{m-1} \sum_{i_{1}, i_{2}, \ldots, i_{m-1}=1}^{2} \frac{1}{k_{i_{1} i_{2 r_{1}} \ldots i_{m-1} r_{m-2}}} X_{i_{1} i_{2} \ldots i_{m-1}}\right. \\
\left.\times u_{i_{1}}^{\left(m-r_{1}-(m-3)\right)} \ldots u_{i_{m-1}}^{\left(r_{1}-r_{2}-\ldots-r_{m-2}+(m-3)\right)}\right) \\
+\mathcal{C}_{m} \sum_{i_{1}, i_{2}, \ldots, i_{m}=1}^{2} \frac{1}{m !} X_{i_{1} i_{2} \ldots i_{m}} u_{i_{1}}^{\prime} u_{i_{2}}^{\prime} \ldots u_{i_{m}}^{\prime}, \quad m \geq 2,
\end{array}
$$

where

$$
\begin{aligned}
\mathcal{C}_{1} & =\left(\begin{array}{c}
m \\
m
\end{array}\right), \quad \mathcal{C}_{2}=\left(\begin{array}{c}
m \\
m-r_{1}
\end{array}\right)\left(\begin{array}{c}
r_{1} \\
r_{1}
\end{array}\right), \\
\mathcal{C}_{3} & =\left(\begin{array}{c}
m \\
m-r_{1}-1
\end{array}\right)\left(\begin{array}{c}
r_{1}+1 \\
r_{2}
\end{array}\right)\left(\begin{array}{c}
r_{1}-r_{2}+1 \\
r_{1}-r_{2}+1
\end{array}\right), \\
\mathcal{C}_{4} & =\left(\begin{array}{c}
m_{m} \\
m-r_{1}-2
\end{array}\right)\left(\begin{array}{c}
r_{1}+2 \\
r_{2}
\end{array}\right)\left(\begin{array}{c}
r_{1}-r_{2}+2 \\
r_{3}
\end{array}\right)\left(\begin{array}{c}
r_{1}-r_{2}-r_{3}+2 \\
r_{1}-r_{2}-r_{3}+2
\end{array}\right), \\
& \vdots \\
\mathcal{C}_{m} & =\left(\begin{array}{c}
m \\
1
\end{array}\right)\left(\begin{array}{c}
m-1 \\
1
\end{array}\right)\left(\begin{array}{c}
m-2 \\
1
\end{array}\right) \ldots\left(\begin{array}{l}
1 \\
1
\end{array}\right), \quad\left(\begin{array}{llll}
\text { each } & \mathcal{C}_{\ell} & \text { includes } \quad \ell & \text { combinations })
\end{array}\right.
\end{aligned}
$$

the factors $\mathcal{C}_{i}, 1<i<m$, are determined by

$$
\left(\begin{array}{c}
m \\
m-r_{1}-(i-2)
\end{array}\right)\left(\begin{array}{c}
r_{1}+i-2 \\
r_{2}
\end{array}\right) \cdots\left(\begin{array}{c}
r_{1}-r_{2}-\ldots-r_{i-2}+i-2 \\
r_{i-1}
\end{array}\right)\left(\begin{array}{c}
r_{1}-r_{2}-\ldots-r_{i-1}+i-2 \\
r_{1}-r_{2}-\ldots-r_{i-1}+i-2
\end{array}\right),
$$

the terms $b$ of each $\left(\begin{array}{l}a \\ b\end{array}\right)$ represents the exponent of $u_{i_{j}}$ in which the terms $u_{i_{1}}^{\left(t_{1}\right)}, u_{i_{2}}^{\left(t_{2}\right)}, u_{i_{3}}^{\left(t_{3}\right)}, u_{i_{4}}^{\left(t_{4}\right)}, u_{i_{5}}^{\left(t_{5}\right)}, \ldots, u_{i_{m}}^{\left(t_{m}\right)}$ are written in the order such that $t_{1} \geq$ $t_{2} \geq t_{3} \geq \ldots \geq t_{m}$, and if $t_{i} \leq 0,1 \leq i \leq m$, the related sum term is canceled (see appendix $A$ for explanation). The coefficients $k_{i_{1}}, k_{i_{1} i_{r_{1}}}$, $k_{i_{1} i_{r_{1}} i_{3 r_{2}} i_{4 r_{3}}} \ldots, k_{i_{1} i_{2_{1}} \ldots i_{m-1} r_{m-2}}$ are calculated as shown in Table 1, where the integer $q$ in each $q$ ! represents the number of repeated exponents.

\begin{tabular}{|c|c|}
\hline & $k_{i_{1}}, k_{i_{1} i_{2} r_{1}}, \ldots, k_{i_{1} i_{2 r_{1}} \ldots i_{m-1} r_{m-2}}$ \\
\hline$u_{i_{1}}^{\left(t_{1}\right)} u_{i_{2}}^{\left(t_{2}\right)} u_{i_{3}}^{\left(t_{3}\right)} u_{i_{4}}^{\left(t_{4}\right)} u_{i_{5}}^{\left(t_{5}\right)} \ldots u_{i_{m}}^{\left(t_{m}\right)}$ & $1 !$ \\
$u_{i_{1}}^{\left(t_{1}\right)} u_{i_{2}}^{\left(t_{1}\right)} u_{i_{3}}^{\left(t_{3}\right)} u_{\left.i_{4}\right)}^{\left(t_{4}\right)} u_{\left.i_{5}\right)}^{\left(t_{5}\right)} \ldots u_{\left.i_{m}\right)}^{\left(t_{m}\right)}$ & $2 !$ \\
$u_{i_{1}}^{\left(t_{1}\right)} u_{i_{2}}^{\left(t_{1}\right)} u_{i_{3}}^{\left(t_{2}\right)} u_{i_{4}}^{\left(t_{2}\right)} u_{\left.i_{5}\right)}^{\left(t_{5}\right)} \ldots u_{i_{m}}^{\left(t_{m}\right)}$ & $2 ! 2 !$ \\
$u_{i_{1}}^{\left(t_{1}\right)} u_{i_{2}}^{\left(t_{1}\right)} u_{i_{3}}^{\left(t_{1}\right)} u_{i_{4}}^{\left(t_{4}\right)} u_{i_{5}}^{\left(t_{5}\right)} \ldots u_{\left.i_{m}\right)}^{\left(t_{m}\right)}$ & $3 !$ \\
$u_{i_{1}}^{\left(t_{1}\right)} u_{i_{2}}^{\left(t_{1}\right)} u_{i_{3}}^{\left(t_{1}\right)} u_{i_{4}}^{\left(t_{2}\right)} u_{i_{5}}^{\left(t_{2}\right)} \ldots u_{i_{m}}^{\left(t_{m}\right)}$ & $3 ! 2 !$ \\
& $\vdots$ \\
& $\vdots$ \\
$u_{i_{1}}^{\left(t_{1}\right)} u_{i_{2}}^{\left(t_{1}\right)} u_{i_{3}}^{\left(t_{1}\right)} u_{i_{4}}^{\left(t_{1}\right)} u_{i_{5}}^{\left(t_{1}\right)} \ldots u_{i_{m}}^{\left(t_{1}\right)}$ & $\vdots$ \\
\hline
\end{tabular}

TABLE 1. Determination of $k_{i_{1}}, k_{i_{1} i_{2_{1}}}, k_{i_{1} i_{2_{1}} i_{3 r_{2}} i_{4 r_{3}}} \ldots, k_{i_{1} i_{2 r_{1}} \ldots i_{m-1} r_{r_{m-2}}}$.

Proof. Let us prove it by induction. 
Step I: Let us show that it is true for $m=2$. In this case, $r_{1}=1$. Then, we may write

$$
\begin{gathered}
\alpha^{\prime \prime}(s)=\left(\begin{array}{l}
2 \\
2
\end{array}\right) \sum_{i_{1}=1}^{2} \frac{1}{k_{i_{1}}} X_{i_{1}} u_{i_{1}}^{\prime \prime}+\left(\begin{array}{l}
2 \\
1
\end{array}\right)\left(\begin{array}{l}
1 \\
1
\end{array}\right) \sum_{i_{1}, i_{2}=1}^{2} \frac{1}{k_{i_{1} i_{1}}} X_{i_{1} i_{2}} u_{i_{1}}^{\prime} u_{i_{2}}^{\prime} \\
=\frac{1}{k_{1}} X_{1} u_{1}^{\prime \prime}+\frac{1}{k_{2}} X_{2} u_{2}^{\prime \prime}+2 \frac{1}{k_{11_{1}}} X_{11} u_{1}^{\prime} u_{1}^{\prime}+2 \frac{1}{k_{12_{1}}} X_{12} u_{1}^{\prime} u_{2}^{\prime} \\
+2 \frac{1}{k_{21_{1}}} X_{21} u_{2}^{\prime} u_{1}^{\prime}+2 \frac{1}{k_{22_{1}}} X_{22} u_{2}^{\prime} u_{2}^{\prime} .
\end{gathered}
$$

From Table 1 given above, we have $k_{1}=k_{2}=1, k_{11_{1}}=k_{12_{1}}=k_{21_{1}}=k_{22_{1}}=$ 2 , which yields

$$
\begin{aligned}
\alpha^{\prime \prime}(s) & =X_{1} u_{1}^{\prime \prime}+X_{2} u_{2}^{\prime \prime}+X_{11} u_{1}^{\prime} u_{1}^{\prime}+X_{12} u_{1}^{\prime} u_{2}^{\prime}+X_{21} u_{2}^{\prime} u_{1}^{\prime}+X_{22} u_{2}^{\prime} u_{2}^{\prime} \\
& =\sum_{i_{1}=1}^{2} X_{i_{1}} u_{i_{1}}^{\prime \prime}+\sum_{i_{1}, i_{2}=1}^{2} X_{i_{1} i_{2}} u_{i_{1}}^{\prime} u_{i_{2}}^{\prime}
\end{aligned}
$$

as given in (2.2). Thus, the statement holds for $m=2$.

Step II: We assume that the formula (3.1) is true for $m$.

Step III: Let us show that the formula is true for $m+1$. By taking the derivative of both sides of $\alpha^{(m)}$, we obtain

$$
\begin{aligned}
& \alpha^{(m+1)}=\mathcal{C}_{1}\left[\sum_{i_{1}, i_{2}=1}^{2} \frac{1}{k_{i_{1}}} X_{i_{1} i_{2}} u_{i_{1}}^{(m)} u_{i_{2}}^{\prime}+\sum_{i_{1}=1}^{2} \frac{1}{k_{i_{1}}} X_{i_{1}} u_{i_{1}}^{(m+1)}\right] \\
& +\sum_{r_{1}=1}^{\left\lfloor\frac{m}{2}\right\rfloor} \mathcal{C}_{2}\left[\sum_{i_{1}, i_{2}, i_{3}=1}^{2} \frac{1}{k_{i_{1} i_{2 r_{1}}}} X_{i_{1} i_{2} i_{3}} u_{i_{1}}^{\left(m-r_{1}\right)} u_{i_{2}}^{\left(r_{1}\right)} u_{i_{3}}^{\prime}\right. \\
& \left.+\sum_{i_{1}, i_{2}=1}^{2} \frac{1}{k_{i_{1} i_{2_{1}}}} X_{i_{1} i_{2}} u_{i_{1}}^{\left(m-r_{1}+1\right)} u_{i_{2}}^{\left(r_{1}\right)}+\sum_{i_{1}, i_{2}=1}^{2} \frac{1}{k_{i_{1} i_{2}}} X_{i_{1} i_{2}} u_{i_{1}}^{\left(m-r_{1}\right)} u_{i_{2}}^{\left(r_{1}+1\right)}\right] \\
& +\sum_{r_{1}, r_{2}=1}^{\left\lfloor\frac{m}{2}\right\rfloor} \mathcal{C}_{3}\left[\sum_{i_{1}, i_{2}, i_{3}, i_{4}=1}^{2} \frac{1}{k_{i_{1} i_{2 r_{1}} i_{3 r_{2}}}} X_{i_{1} i_{2} i_{3} i_{4}} u_{i_{1}}^{\left(m-r_{1}-1\right)} u_{i_{2}}^{\left(r_{2}\right)} u_{i_{3}}^{\left(r_{1}-r_{2}+1\right)} u_{i_{4}}^{\prime}\right. \\
& +\sum_{i_{1}, i_{2}, i_{3}=1}^{2} \frac{1}{k_{i_{1} i_{2 r_{1}} i_{3 r_{2}}}} X_{i_{1} i_{2} i_{3}} u_{i_{1}}^{\left(m-r_{1}\right)} u_{i_{2}}^{\left(r_{2}\right)} u_{i_{3}}^{\left(r_{1}-r_{2}+1\right)} \\
& +\sum_{i_{1}, i_{2}, i_{3}=1}^{2} \frac{1}{k_{i_{1} i_{2 r_{1}} i_{3 r_{2}}}} X_{i_{1} i_{2} i_{3}} u_{i_{1}}^{\left(m-r_{1}-1\right)} u_{i_{2}}^{\left(r_{2}+1\right)} u_{i_{3}}^{\left(r_{1}-r_{2}+1\right)} \\
& \left.+\sum_{i_{1}, i_{2}, i_{3}=1}^{2} \frac{1}{k_{i_{1} i_{2 r_{1}} i_{3 r_{2}}}} X_{i_{1} i_{2} i_{3}} u_{i_{1}}^{\left(m-r_{1}-1\right)} u_{i_{2}}^{\left(r_{2}\right)} u_{i_{3}}^{\left(r_{1}-r_{2}+2\right)}\right]
\end{aligned}
$$




$$
\begin{aligned}
&+\mathcal{C}_{m}\left[\sum_{i_{1}, i_{2}, \ldots, i_{m+1}=1}^{2} \frac{1}{m !} X_{i_{1} i_{2} \ldots i_{m+1}} u_{i_{1}}^{\prime} u_{i_{2}}^{\prime} \ldots u_{i_{m+1}}^{\prime}\right. \\
&\left.+m \sum_{i_{1}, i_{2}, \ldots, i_{m}=1}^{2} \frac{1}{m !} X_{i_{1} i_{2} \ldots i_{m}} u_{i_{1}}^{\prime \prime} u_{i_{2}}^{\prime} \ldots u_{i_{m}}^{\prime}\right]
\end{aligned}
$$

or

$$
\begin{aligned}
& \alpha^{(m+1)}=\underbrace{\mathcal{C}_{1} \sum_{i_{1}=1}^{2} \frac{1}{k_{i_{1}}} X_{i_{1}} u_{i_{1}}^{(m+1)}}_{1} \\
& +\mathcal{C}_{1} \sum_{i_{1}, i_{2}=1}^{2} \frac{1}{k_{i_{1}}} X_{i_{1} i_{2}} u_{i_{1}}^{(m)} u_{i_{2}}^{\prime}
\end{aligned}
$$

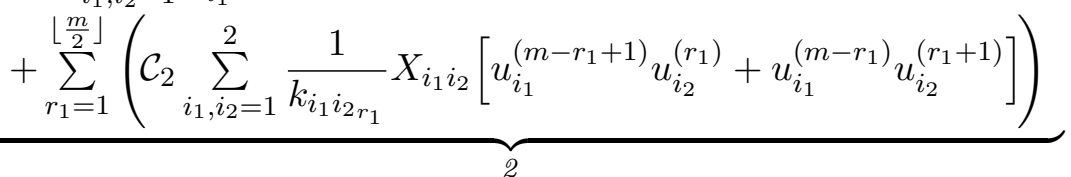

$$
\begin{aligned}
& +\sum_{r_{1}=1}^{\left\lfloor\frac{m}{2}\right\rfloor}\left(\mathcal{C}_{2} \sum_{i_{1}, i_{2}, i_{3}=1}^{2} \frac{1}{k_{i_{1} i_{2 r_{1}}}} X_{i_{1} i_{2} i_{3}} u_{i_{1}}^{\left(m-r_{1}\right)} u_{i_{2}}^{\left(r_{1}\right)} u_{i_{3}}^{\prime}\right) \\
& +\sum_{r_{1}, r_{2}=1}^{\left\lfloor\frac{m}{2}\right\rfloor}\left(\mathcal { C } _ { 3 } \sum _ { i _ { 1 } , i _ { 2 } , i _ { 3 } = 1 } ^ { 2 } \frac { 1 } { k _ { i _ { 1 } i _ { 2 r _ { 1 } } i _ { 3 r _ { 2 } } } } X _ { i _ { 1 } i _ { 2 } i _ { 3 } } \left[u_{i_{1}}^{\left(m-r_{1}\right)} u_{i_{2}}^{\left(r_{2}\right)} u_{i_{3}}^{\left(r_{1}-r_{2}+1\right)}\right.\right. \\
& \left.\left.+u_{i_{1}}^{\left(m-r_{1}-1\right)} u_{i_{2}}^{\left(r_{2}+1\right)} u_{i_{3}}^{\left(r_{1}-r_{2}+1\right)}\right]\right)
\end{aligned}
$$

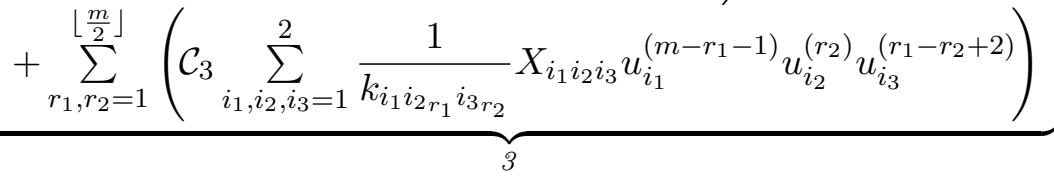

$$
\begin{aligned}
& \underbrace{\mathcal{C}_{m} \sum_{i_{1}, i_{2}, \ldots, i_{m+1}=1}^{2} \frac{1}{m !} X_{i_{1} i_{2} \ldots i_{m+1}} u_{i_{1}}^{\prime} u_{i_{2}}^{\prime} \ldots u_{i_{m+1}}^{\prime}}_{m+1} .
\end{aligned}
$$

If we consider the values of $r_{i}, r_{i} \in\left[1,\left\lfloor\frac{m}{2}\right\rfloor\right]$, the sums in $1,2,3, \ldots, m+1$ given above can be expressed as a single sum as follows:

$$
\begin{array}{ll}
\text { 1: } & \left(\begin{array}{c}
m+1 \\
m+1
\end{array}\right) \sum_{i_{1}=1}^{2} \frac{1}{k_{i_{1}}} X_{i_{1}} u_{i_{1}}^{(m+1)} . \\
\text { 2: } \quad & \sum_{r_{1}=1}^{\left\lfloor\frac{m+1}{2}\right\rfloor}\left(\left(\begin{array}{c}
m+1 \\
m+1-r_{1}
\end{array}\right) \sum_{i_{1}, i_{2}=1}^{2} \frac{1}{k_{i_{1} i_{2} r_{1}}} X_{i_{1} i_{2}} u_{i_{1}}^{\left(m-r_{1}+1\right)} u_{i_{2}}^{\left(r_{1}\right)}\right) .
\end{array}
$$




$$
\begin{array}{cc}
\text { 3: } & \sum_{r_{1}, r_{2}=1}^{\left\lfloor\frac{m+1}{2}\right\rfloor}\left(\left(\begin{array}{c}
m+1 \\
m-r_{1}
\end{array}\right)\left(\begin{array}{c}
r_{1}+1 \\
r_{2}
\end{array}\right) \sum_{i_{1}, i_{2}, i_{3}=1}^{2} \frac{1}{k_{i_{1} i_{2} i_{1} i_{3}}} X_{i_{1} i_{2} i_{3}} u_{i_{1}}^{\left(m-r_{1}\right)} u_{i_{2}}^{\left(r_{2}\right)} u_{i_{3}}^{\left(r_{1}-r_{2}+1\right)}\right) . \\
\vdots & \\
m+1: & \left(\begin{array}{c}
m+1 \\
1
\end{array}\right)\left(\begin{array}{c}
m \\
1
\end{array}\right)\left(\begin{array}{c}
m-1 \\
1
\end{array}\right) \ldots\left(\begin{array}{l}
1 \\
1
\end{array}\right) \sum_{i_{1}, i_{2}, \ldots, i_{m+1}=1}^{2} \frac{1}{(m+1) !} X_{i_{1} i_{2} \ldots i_{m+1}} u_{i_{1}}^{\prime} u_{i_{2}}^{\prime} \ldots u_{i_{m+1}}^{\prime} .
\end{array}
$$

Then, the sum $1+2+3+\ldots+m+1$ gives us the result.

\section{Transversal intersection curve of two parametric surfaces in $\mathbb{E}^{n}$}

Let $\mathcal{S}_{1}$ and $\mathcal{S}_{2}$ be regular intersecting surfaces given by their parametric equations

$$
X\left(u_{1}, u_{2}\right)=\left(f_{1}\left(u_{1}, u_{2}\right), f_{2}\left(u_{1}, u_{2}\right), \ldots, f_{n}\left(u_{1}, u_{2}\right)\right)
$$

and

$$
Y\left(w_{1}, w_{2}\right)=\left(g_{1}\left(w_{1}, w_{2}\right), g_{2}\left(w_{1}, w_{2}\right), \ldots, g_{n}\left(w_{1}, w_{2}\right)\right)
$$

in $\mathbb{E}^{n}, n \geq 4$, respectively. Then, the tangent spaces of $\mathcal{S}_{1}$ and $\mathcal{S}_{2}$ are spanned by $\left\{X_{u_{1}}, X_{u_{2}}\right\}$ and $\left\{Y_{w_{1}}, Y_{w_{2}}\right\}$, respectively. Let $\alpha(s)$ be their intersection curve with arc-length parameter $s$.

Let us consider the set

$$
W=\left\{X_{u_{1}}, X_{u_{2}}, Y_{w_{1}}, Y_{w_{2}}\right\} .
$$

For the tangent vector fields in $W$ we have the following cases at an intersection point $\alpha(s)$.

Case 1. If $W$ is linearly independent, then $\alpha(s)$ is an isolated point of such intersection.

Case 2. If $W$ has only two linearly independent vectors at an intersection point, then the tangent spaces of $\mathcal{S}_{1}$ and $\mathcal{S}_{2}$ overlap at that point. This kind of intersection is called a tangential intersection. We exclude such intersections.

Case 3. If $W$ has three linearly independent vectors at an intersection point, then the tangent spaces of $\mathcal{S}_{1}$ and $\mathcal{S}_{2}$ differ at that point. This kind of intersection is called a transversal intersection. We focus on such intersections.

Let us consider the case 3 and denote the Frenet frame of $\alpha$ by $\left\{\mathbf{V}_{1}\right.$, $\left.\mathbf{V}_{2}, \ldots, \mathbf{V}_{n}\right\}$ at the intersection point $P=X\left(u_{1}(0), u_{2}(0)\right)=Y\left(w_{1}(0), w_{2}(0)\right)$. 
4.1. Tangent vector $\left(\alpha^{\prime}\right)$. We need bases of the normal spaces of $\mathcal{S}_{1}$ and $\mathcal{S}_{2}$ to obtain the tangent vector $\mathbf{V}_{1}$. To obtain a basis of the normal space of $\mathcal{S}_{1}$, we choose $n-3$ vectors from the standart basis $\left\{\mathbf{e}_{1}, \mathbf{e}_{2}, \ldots, \mathbf{e}_{n}\right\}$ of $\mathbb{R}^{n}$ to be linearly independent with $\left\{X_{u_{1}}, X_{u_{2}}\right\}$. Without loss of generality, we assume that $\left\{X_{u_{1}}, X_{u_{2}}, \mathbf{e}_{1}, \mathbf{e}_{2}, \ldots, \mathbf{e}_{n-3}\right\}$ is linearly independent at $P$. Let us define

$$
\begin{aligned}
\mathbf{N}_{1}^{X} & =X_{u_{1}} \times X_{u_{2}} \times \mathbf{e}_{1} \times \mathbf{e}_{2} \times \ldots \times \mathbf{e}_{n-3}, \\
\mathbf{N}_{2}^{X} & =X_{u_{1}} \times X_{u_{2}} \times \mathbf{N}_{1}^{X} \times \mathbf{e}_{1} \times \ldots \times \mathbf{e}_{n-4}, \\
\mathbf{N}_{3}^{X} & =X_{u_{1}} \times X_{u_{2}} \times \mathbf{N}_{1}^{X} \times \mathbf{N}_{2}^{X} \times \mathbf{e}_{1} \times \ldots \times \mathbf{e}_{n-5}, \\
& \vdots \\
\mathbf{N}_{n-2}^{X} & =X_{u_{1}} \times X_{u_{2}} \times \mathbf{N}_{1}^{X} \times \mathbf{N}_{2}^{X} \times \ldots \times \mathbf{N}_{n-3}^{X} .
\end{aligned}
$$

It is clear from the above equations that $\left\{\mathbf{N}_{1}^{X}, \mathbf{N}_{2}^{X}, \ldots, \mathbf{N}_{n-2}^{X}\right\}$ constitutes an orthogonal basis for the normal space of $\mathcal{S}_{1}$ at $P$. Similarly, assuming that $\left\{Y_{w_{1}}, Y_{w_{2}}, \mathbf{e}_{1}, \mathbf{e}_{2}, \ldots, \mathbf{e}_{n-3}\right\}$ is linearly independent at $P$ and defining

$$
\begin{aligned}
\mathbf{N}_{1}^{Y} & =Y_{w_{1}} \times Y_{w_{2}} \times \mathbf{e}_{1} \times \mathbf{e}_{2} \times \ldots \times \mathbf{e}_{n-3}, \\
\mathbf{N}_{2}^{Y} & =Y_{w_{1}} \times Y_{w_{2}} \times \mathbf{N}_{1}^{Y} \times \mathbf{e}_{1} \times \ldots \times \mathbf{e}_{n-4}, \\
\mathbf{N}_{3}^{Y} & =Y_{w_{1}} \times Y_{w_{2}} \times \mathbf{N}_{1}^{Y} \times \mathbf{N}_{2}^{Y} \times \mathbf{e}_{1} \times \ldots \times \mathbf{e}_{n-5}, \\
& \vdots \\
\mathbf{N}_{n-2}^{Y}= & Y_{w_{1}} \times Y_{w_{2}} \times \mathbf{N}_{1}^{Y} \times \mathbf{N}_{2}^{Y} \times \ldots \times \mathbf{N}_{n-3}^{Y},
\end{aligned}
$$

yields the orthogonal basis $\left\{\mathbf{N}_{1}^{Y}, \mathbf{N}_{2}^{Y}, \ldots, \mathbf{N}_{n-2}^{Y}\right\}$ for the normal space of $\mathcal{S}_{2}$ at $P$. Let

$$
\mathcal{N}=\left\{\mathbf{N}_{1}^{X}, \mathbf{N}_{2}^{X}, \ldots, \mathbf{N}_{n-2}^{X}, \mathbf{N}_{1}^{Y}, \mathbf{N}_{2}^{Y}, \ldots, \mathbf{N}_{n-2}^{Y}\right\}
$$

and $d$ be the dimension of the subspace spanned by $\mathcal{N}$. It is clear that the value of $d$ can be $n-2, n-1$ or $n$. These values of $d$ correspond to the following intersection types:

a) If $d=n$, then the intersection point $P$ is an isolated point.

b) If $d=n-2$, then the normal spaces of the surfaces $\mathcal{S}_{1}$ and $\mathcal{S}_{2}$ overlap at $P$, i.e. we have a tangential intersection.

c) If $d=n-1$, then we have a transversal intersection at $P$.

Since we consider the transversal intersection, let us assume that $d=n-1$ and $\left\{\mathbf{N}_{1}^{X}, \mathbf{N}_{2}^{X}, \ldots, \mathbf{N}_{n-2}^{X}, \mathbf{N}_{\ell}^{Y}\right\}, \ell \in\{1,2, \ldots, n-2\}$ is linearly independent at $P$. Since $\mathbf{V}_{1} \perp \mathbf{N}_{j}^{X}, 1 \leq j \leq n-2$ and $\mathbf{V}_{1} \perp \mathbf{N}_{\ell}^{Y}$, the tangent vector of the intersection curve can be obtained by

$$
\mathbf{V}_{1}=\frac{\mathbf{N}_{1}^{X} \times \mathbf{N}_{2}^{X} \times \ldots \times \mathbf{N}_{n-2}^{X} \times \mathbf{N}_{\ell}^{Y}}{\left\|\mathbf{N}_{1}^{X} \times \mathbf{N}_{2}^{X} \times \ldots \times \mathbf{N}_{n-2}^{X} \times \mathbf{N}_{\ell}^{Y}\right\|} .
$$

Thus, if we take the dot product of both sides of

$$
\mathbf{V}_{1}=X_{u_{1}} u_{1}^{\prime}+X_{u_{2}} u_{2}^{\prime}
$$


with $X_{u_{1}}, X_{u_{2}}$ and of

$$
\mathbf{V}_{1}=Y_{w_{1}} w_{1}^{\prime}+Y_{w_{2}} w_{2}^{\prime}
$$

with $Y_{w_{1}}, Y_{w_{2}}$, the coefficients $u_{1}^{\prime}, u_{2}^{\prime}$ and $w_{1}^{\prime}, w_{2}^{\prime}$ can be found by

$$
u_{1}^{\prime}=\frac{G\left\langle\mathbf{V}_{1}, X_{u_{1}}\right\rangle-F\left\langle\mathbf{V}_{1}, X_{u_{2}}\right\rangle}{E G-F^{2}}, \quad u_{2}^{\prime}=\frac{E\left\langle\mathbf{V}_{1}, X_{u_{2}}\right\rangle-F\left\langle\mathbf{V}_{1}, X_{u_{1}}\right\rangle}{E G-F^{2}}
$$

and

$$
w_{1}^{\prime}=\frac{g\left\langle\mathbf{V}_{1}, Y_{w_{1}}\right\rangle-f\left\langle\mathbf{V}_{1}, Y_{w_{2}}\right\rangle}{e g-f^{2}}, \quad w_{2}^{\prime}=\frac{e\left\langle\mathbf{V}_{1}, Y_{w_{2}}\right\rangle-f\left\langle\mathbf{V}_{1}, Y_{w_{1}}\right\rangle}{e g-f^{2}},
$$

where $E, F, G$ and $e, f, g$ are the first fundamental form coefficients of $\mathcal{S}_{1}$ and $\mathcal{S}_{2}$, respectively.

4.2. Second derivative $\left(\alpha^{\prime \prime}\right)$. Since $\alpha^{\prime \prime}$ is orthogonal to $\mathbf{V}_{1}$, we may write

$$
\alpha^{\prime \prime}=\lambda_{1} \mathbf{N}_{1}^{X}+\lambda_{2} \mathbf{N}_{2}^{X}+\ldots+\lambda_{n-2} \mathbf{N}_{n-2}^{X}+\lambda_{n-1} \mathbf{N}_{\ell}^{Y} .
$$

We must determine the coefficients $\lambda_{i}, 1 \leq i \leq n-1$. If we take the dot product of both sides of (4.4) with $\mathbf{N}_{1}^{X}, \mathbf{N}_{2}^{X}, \ldots, \mathbf{N}_{n-2}^{X}, \mathbf{N}_{\ell}^{Y}$, respectively, we obtain the system of linear equations depending on $\lambda_{i}, 1 \leq i \leq n-1$, as

$$
\begin{gathered}
\left\langle\mathbf{N}_{1}^{X}, \mathbf{N}_{1}^{X}\right\rangle \lambda_{1}+\left\langle\mathbf{N}_{\ell}^{Y}, \mathbf{N}_{1}^{X}\right\rangle \lambda_{n-1}=\left\langle\alpha^{\prime \prime}, \mathbf{N}_{1}^{X}\right\rangle \\
\left\langle\mathbf{N}_{2}^{X}, \mathbf{N}_{2}^{X}\right\rangle \lambda_{2}+\left\langle\mathbf{N}_{\ell}^{Y}, \mathbf{N}_{2}^{X}\right\rangle \lambda_{n-1}=\left\langle\alpha^{\prime \prime}, \mathbf{N}_{2}^{X}\right\rangle \\
\vdots \\
\left\langle\mathbf{N}_{n-2}^{X}, \mathbf{N}_{n-2}^{X}\right\rangle \lambda_{n-2}+\left\langle\mathbf{N}_{\ell}^{Y}, \mathbf{N}_{n-2}^{X}\right\rangle \lambda_{n-1}=\left\langle\alpha^{\prime \prime}, \mathbf{N}_{n-2}^{X}\right\rangle \\
\left\langle\mathbf{N}_{1}^{X}, \mathbf{N}_{\ell}^{Y}\right\rangle \lambda_{1}+\left\langle\mathbf{N}_{2}^{X}, \mathbf{N}_{\ell}^{Y}\right\rangle \lambda_{2}+\ldots+\left\langle\mathbf{N}_{\ell}^{Y}, \mathbf{N}_{\ell}^{Y}\right\rangle \lambda_{n-1}=\left\langle\alpha^{\prime \prime}, \mathbf{N}_{\ell}^{Y}\right\rangle .
\end{gathered}
$$

For the coefficient determinant

$$
\operatorname{det} \mathcal{A}=\left|\begin{array}{ccccc}
\left\langle\mathbf{N}_{1}^{X}, \mathbf{N}_{1}^{X}\right\rangle & 0 & \cdots & 0 & \left\langle\mathbf{N}_{\ell}^{Y}, \mathbf{N}_{1}^{X}\right\rangle \\
0 & \left\langle\mathbf{N}_{2}^{X}, \mathbf{N}_{2}^{X}\right\rangle & \cdots & 0 & \left\langle\mathbf{N}_{\ell}^{Y}, \mathbf{N}_{2}^{X}\right\rangle \\
\vdots & \vdots & & \vdots & \\
0 & 0 & \cdots & \left\langle\mathbf{N}_{n-2}^{X}, \mathbf{N}_{n-2}^{X}\right\rangle & \left\langle\mathbf{N}_{\ell}^{Y}, \mathbf{N}_{n-2}^{X}\right\rangle \\
\left\langle\mathbf{N}_{1}^{X}, \mathbf{N}_{\ell}^{Y}\right\rangle & \left\langle\mathbf{N}_{2}^{X}, \mathbf{N}_{\ell}^{Y}\right\rangle & \cdots & \left\langle\mathbf{N}_{n-2}^{X}, \mathbf{N}_{\ell}^{Y}\right\rangle & \left\langle\mathbf{N}_{\ell}^{Y}, \mathbf{N}_{\ell}^{Y}\right\rangle
\end{array}\right|
$$

of this system, by using Definition 1 we have

$$
\operatorname{det} \mathcal{A}=\left\|\mathbf{N}_{1}^{X} \times \mathbf{N}_{2}^{X} \times \cdots \times \mathbf{N}_{n-2}^{X} \times \mathbf{N}_{\ell}^{Y}\right\|^{2} \neq 0 .
$$

Using the Cramer's method, the solution of this system is then obtained as

$$
\lambda_{i}=\frac{1}{\operatorname{det} \mathcal{A}}\left\{\left\langle\alpha^{\prime \prime}, \mathbf{N}_{\ell}^{Y}\right\rangle \mathcal{A}_{(n-1) i}+\sum_{j=1}^{n-2}\left\langle\alpha^{\prime \prime}, \mathbf{N}_{j}^{X}\right\rangle \mathcal{A}_{j i}\right\}, \quad 1 \leq i \leq n-1,
$$


where $\mathcal{A}_{j i}$ denotes the $(j, i)$ cofactor of the matrix $\mathcal{A}$. Besides, if we use (2.2), we have

$$
\begin{aligned}
& \left\langle\alpha^{\prime \prime}, \mathbf{N}_{j}^{X}\right\rangle=\sum_{i_{1}, i_{2}=1}^{2}\left\langle X_{i_{1} i_{2}}, \mathbf{N}_{j}^{X}\right\rangle u_{i_{1}}^{\prime} u_{i_{2}}^{\prime}, \\
& \left\langle\alpha^{\prime \prime}, \mathbf{N}_{\ell}^{Y}\right\rangle=\sum_{i_{1}, i_{2}=1}^{2}\left\langle Y_{i_{1} i_{2}}, \mathbf{N}_{\ell}^{Y}\right\rangle w_{i_{1}}^{\prime} w_{i_{2}}^{\prime} .
\end{aligned}
$$

Since the right sides of the equations in (4.6) are known, substitution of (4.6) into (4.5) yields $\lambda_{i}$, which allows us to compute $\alpha^{\prime \prime}$ by using (4.4). Hence, $u_{1}^{\prime \prime}, u_{2}^{\prime \prime}$ and $w_{1}^{\prime \prime}, w_{2}^{\prime \prime}$ can be obtained by using (2.2). Also, using the Gram-Schmidt orthogonalization method, we find

$$
\mathbf{E}_{2}=\alpha^{\prime \prime}-\left\langle\alpha^{\prime \prime}, \mathbf{V}_{1}\right\rangle \mathbf{V}_{1}, \quad \mathbf{V}_{2}=\frac{\mathbf{E}_{2}}{\left\|\mathbf{E}_{2}\right\|} .
$$

Then, since $\alpha^{\prime \prime}=k_{1} \mathbf{V}_{2}$, the first curvature of the intersection curve is obtained by $k_{1}=\left\langle\alpha^{\prime \prime}, \mathbf{V}_{2}\right\rangle$.

4.3. Third derivative $\left(\alpha^{\prime \prime \prime}\right)$. For the third derivative of the intersection curve, we may write

$$
\begin{aligned}
\alpha^{\prime \prime \prime} & =-k_{1}^{2} \mathbf{V}_{1}+k_{1}^{\prime} \mathbf{V}_{2}+k_{1} k_{2} \mathbf{V}_{3} \\
& =-k_{1}^{2} \mathbf{V}_{1}+\mu_{1} \mathbf{N}_{1}^{X}+\mu_{2} \mathbf{N}_{2}^{X}+\ldots+\mu_{n-2} \mathbf{N}_{n-2}^{X}+\mu_{n-1} \mathbf{N}_{\ell}^{Y} .
\end{aligned}
$$

Similarly, by taking the dot product of both sides of (4.8) with $\mathbf{N}_{1}^{X}, \mathbf{N}_{2}^{X}, \ldots$, $\mathbf{N}_{n-2}^{X}, \mathbf{N}_{\ell}^{Y}$, respectively, we obtain a system of linear equations depending on $\mu_{i}$ which has the solution

$$
\mu_{i}=\frac{1}{\operatorname{det} \mathcal{A}}\left\{\left\langle\alpha^{\prime \prime \prime}, \mathbf{N}_{\ell}^{Y}\right\rangle \mathcal{A}_{(n-1) i}+\sum_{j=1}^{n-2}\left\langle\alpha^{\prime \prime \prime}, \mathbf{N}_{j}^{X}\right\rangle \mathcal{A}_{j i}\right\}, 1 \leq i \leq n-1 .
$$

If we use (2.3), we have

$$
\begin{aligned}
& \left\langle\alpha^{\prime \prime \prime}, \mathbf{N}_{j}^{X}\right\rangle=3 \sum_{i_{1}, i_{2}=1}^{2}\left\langle X_{i_{1} i_{2}}, \mathbf{N}_{j}^{X}\right\rangle u_{i_{1}}^{\prime \prime} u_{i_{2}}^{\prime}+\sum_{i_{1}, i_{2}, i_{3}=1}^{2}\left\langle X_{i_{1} i_{2} i_{3}}, \mathbf{N}_{j}^{X}\right\rangle u_{i_{1}}^{\prime} u_{i_{2}}^{\prime} u_{i_{3}}^{\prime}, \\
& \left\langle\alpha^{\prime \prime \prime}, \mathbf{N}_{\ell}^{Y}\right\rangle=3 \sum_{i_{1}, i_{2}=1}^{2}\left\langle Y_{i_{1} i_{2}}, \mathbf{N}_{\ell}^{Y}\right\rangle w_{i_{1}}^{\prime \prime} w_{i_{2}}^{\prime}+\sum_{i_{1}, i_{2}, i_{3}=1}^{2}\left\langle Y_{i_{1} i_{2} i_{3}}, \mathbf{N}_{\ell}^{Y}\right\rangle w_{i_{1}}^{\prime} w_{i_{2}}^{\prime} w_{i_{3}}^{\prime} .
\end{aligned}
$$

Since the right sides of the equations in (4.10) and (4.11) are known, substitutions of (4.10) and (4.11) into (4.9) yield $\mu_{i}, 1 \leq i \leq n-1$, which enables us to compute $\alpha^{\prime \prime \prime}$ via (4.8). Hence, $u_{1}^{\prime \prime \prime}, u_{2}^{\prime \prime \prime}$ and $w_{1}^{\prime \prime \prime}, w_{2}^{\prime \prime \prime}$ can be found by using (2.3). Using the Gram-Schmidt orthogonalization method, we obtain

$$
\mathbf{E}_{3}=\alpha^{\prime \prime \prime}-\left\langle\alpha^{\prime \prime \prime}, \mathbf{V}_{1}\right\rangle \mathbf{V}_{1}-\left\langle\alpha^{\prime \prime \prime}, \mathbf{V}_{2}\right\rangle \mathbf{V}_{2}, \quad \mathbf{V}_{3}=\frac{\mathbf{E}_{3}}{\left\|\mathbf{E}_{3}\right\|}
$$


Then, if $k_{1} \neq 0$, the second curvature of the intersection curve can be obtained by

$$
k_{2}=\frac{\left\langle\alpha^{\prime \prime \prime}, \mathbf{V}_{3}\right\rangle}{k_{1}}
$$

We also have

$$
k_{1}^{\prime}=\left\langle\alpha^{\prime \prime \prime}, \mathbf{V}_{2}\right\rangle .
$$

4.4. Fourth derivative $\left(\alpha^{(4)}\right)$. Similarly to the second and third derivatives, we may write

$$
\begin{aligned}
\alpha^{(4)} & =-3 k_{1} k_{1}^{\prime} \mathbf{V}_{1}+\left(-k_{1}^{3}+k_{1}^{\prime \prime}-k_{1} k_{2}^{2}\right) \mathbf{V}_{2}+\left(2 k_{1}^{\prime} k_{2}+k_{1} k_{2}^{\prime}\right) \mathbf{V}_{3}+k_{1} k_{2} k_{3} \mathbf{V}_{4}, \\
& =-3 k_{1} k_{1}^{\prime} \mathbf{V}_{1}+\eta_{1} \mathbf{N}_{1}^{X}+\eta_{2} \mathbf{N}_{2}^{X}+\ldots+\eta_{n-2} \mathbf{N}_{n-2}^{X}+\eta_{n-1} \mathbf{N}_{\ell}^{Y},
\end{aligned}
$$

where

$$
\eta_{i}=\frac{1}{\operatorname{det} \mathcal{A}}\left\{\left\langle\alpha^{(4)}, \mathbf{N}_{\ell}^{Y}\right\rangle \mathcal{A}_{(n-1) i}+\sum_{j=1}^{n-2}\left\langle\alpha^{(4)}, \mathbf{N}_{j}^{X}\right\rangle \mathcal{A}_{j i}\right\}
$$

$1 \leq i \leq n-1$, and $\left\langle\alpha^{(4)}, \mathbf{N}_{j}^{X}\right\rangle$ and $\left\langle\alpha^{(4)}, \mathbf{N}_{\ell}^{Y}\right\rangle$ can be obtained by using (2.4). Then, we find

$$
\begin{aligned}
& \mathbf{E}_{4}=\alpha^{(4)}-\left\langle\alpha^{(4)}, \mathbf{V}_{1}\right\rangle \mathbf{V}_{1}-\left\langle\alpha^{(4)}, \mathbf{V}_{2}\right\rangle \mathbf{V}_{2}-\left\langle\alpha^{(4)}, \mathbf{V}_{3}\right\rangle \mathbf{V}_{3}, \\
& \mathbf{V}_{4}=\frac{\mathbf{E}_{4}}{\left\|\mathbf{E}_{4}\right\|} .
\end{aligned}
$$

If $k_{2} \neq 0$, then the third curvature of the intersetion curve is obtained by

$$
k_{3}=\frac{\left\langle\alpha^{(4)}, \mathbf{V}_{4}\right\rangle}{k_{1} k_{2}} .
$$

Also, we obtain $k_{1}^{\prime \prime}$ and $k_{2}^{\prime}$ by using $\left\langle\alpha^{(4)}, \mathbf{V}_{2}\right\rangle$ and $\left\langle\alpha^{(4)}, \mathbf{V}_{3}\right\rangle$, respectively.

4.5. Higher order derivatives $\left(\alpha^{(m)}, m \geq 5\right)$. Similarly, for the $m$ th order derivative of the intersection curve, we can write

$$
\begin{aligned}
\alpha^{(m)} & =d_{1} \mathbf{V}_{1}+d_{2} \mathbf{V}_{2}+\ldots+d_{m-1} \mathbf{V}_{m-1}+k_{1} k_{2} k_{3} \ldots k_{m-1} \mathbf{V}_{m} \\
& =d_{1} \mathbf{V}_{1}+\xi_{1} \mathbf{N}_{1}^{X}+\xi_{2} \mathbf{N}_{2}^{X}+\ldots+\xi_{n-2} \mathbf{N}_{n-2}^{X}+\xi_{n-1} \mathbf{N}_{\ell}^{Y} .
\end{aligned}
$$

We need to determine $\xi_{i}, 1 \leq i \leq n-1$, and only the coefficient $d_{1}$ to find $\alpha^{(m)}$. For $\xi_{i}$, we have

$$
\xi_{i}=\frac{1}{\operatorname{det} \mathcal{A}}\left\{\left\langle\alpha^{(m)}, \mathbf{N}_{\ell}^{Y}\right\rangle \mathcal{A}_{(n-1) i}+\sum_{j=1}^{n-2}\left\langle\alpha^{(m)}, \mathbf{N}_{j}^{X}\right\rangle \mathcal{A}_{j i}\right\}, \quad 1 \leq i \leq n-1,
$$

where $\left\langle\alpha^{(m)}, \mathbf{N}_{j}^{X}\right\rangle$ and $\left\langle\alpha^{(m)}, \mathbf{N}_{\ell}^{Y}\right\rangle$ can be obtained by using (3.1).

On the other hand, as seen in (4.14), for $m \geq 4$ the coefficients $d_{1}$, $d_{2}, \ldots, d_{m-1}$ consist of not only the curvatures $k_{i}$ but also the derivatives 
of these curvatures. However, the curvatures and their derivatives which are included in $d_{1}$ can be obtained by using the previously found Frenet vectors and actual derivatives. For example, $d_{1}$ includes $k_{1}^{\prime}, k_{1}^{\prime \prime}, k_{1}^{\prime \prime \prime}$ and $k_{2}^{\prime}$ for $\alpha^{(6)}$ in which all are known except $k_{1}^{\prime \prime \prime}$. But, $k_{1}^{\prime \prime \prime}$ can be obtained by using $\left\langle\alpha^{(5)}, \mathbf{V}_{2}\right\rangle$. We should note that computing the coefficients $d_{1}, d_{2}, \ldots, d_{m-1}$ manually is difficult and time-consuming for higher order derivatives. To overcome this difficulty, we also provide a code (see appendix B) in MATLAB R2018a which produces all $d_{i}$ for the desired $m$. Thus, we can find $\alpha^{(m)}, m \geq 5$, by using (4.18). If we again use (3.1), we can find $u_{1}^{(m)}, u_{2}^{(m)}, w_{1}^{(m)}, w_{2}^{(m)}$. Also, using the Gram-Schmidt orthogonalization method, we obtain

$$
\mathbf{E}_{m}=\alpha^{(m)}-\sum_{i=1}^{m-1}\left\langle\alpha^{(m)}, \mathbf{V}_{i}\right\rangle \mathbf{V}_{i}, \quad \mathbf{V}_{m}=\frac{\mathbf{E}_{m}}{\left\|\mathbf{E}_{m}\right\|}, \quad 5 \leq m \leq n-1 .
$$

Finally, the last Frenet vector $\mathbf{V}_{n}$ can be obtained by $\mathbf{V}_{n}=\mathbf{V}_{1} \times \mathbf{V}_{2} \times$ $\cdots \times \mathbf{V}_{n-1}$. Hence, if we assume $k_{i} \neq 0, i \geq 3$, the $m$ th curvature of the intersection curve is obtained from

$$
k_{m}=\frac{\left\langle\alpha^{(m)}, \mathbf{V}_{m}\right\rangle}{k_{1} k_{2} \ldots k_{m-1}}, \quad 5 \leq m \leq n-1
$$

Remark 1 . We assume that the curvatures of the intersection curve do not vanish at the intersection point. If any curvature vanishes at an intersection point, we can follow the algorithm given by [21] to obtain the Frenet vectors.

\section{Examples}

5.1. Example in $\mathbb{E}^{4}$. Let us consider the intersection of the Whitney sphere $\mathcal{S}_{1}$ given by the parametrization

$$
\begin{array}{r}
X\left(u_{1}, u_{2}\right)=\frac{1}{1+\left(u_{1}^{2}+u_{2}^{2}\right)^{2}}\left(u_{1}\left(1+u_{1}^{2}+u_{2}^{2}\right), u_{1}\left(u_{1}^{2}+u_{2}^{2}-1\right),\right. \\
\left.u_{2}\left(1+u_{1}^{2}+u_{2}^{2}\right), u_{2}\left(u_{1}^{2}+u_{2}^{2}-1\right)\right),
\end{array}
$$

and the surface $\mathcal{S}_{2}$ given by the parametrization

$$
Y\left(w_{1}, w_{2}\right)=\left(w_{1}, w_{2}, w_{1} w_{2}^{2}, w_{2}^{3}\right)
$$

in $\mathbb{E}^{4}$. These surfaces are projected perspectively [13] from the point $(0,0,0,2)$ into the hyperplane $x_{4}=0$ and their projections are displayed in Figure 1.

Let us find the Frenet vectors and curvatures of their intersection curve $\alpha$ at the intersection point $P=X(1,0)=Y(1,0)=(1,0,0,0)$. The tangent vectors of these surfaces at $P$ are

$$
\begin{aligned}
& X_{u_{1}}=(0,1,0,0), \quad X_{u_{2}}=(0,0,1,0), \\
& Y_{w_{1}}=(1,0,0,0), \quad Y_{w_{2}}=(0,1,0,0) .
\end{aligned}
$$




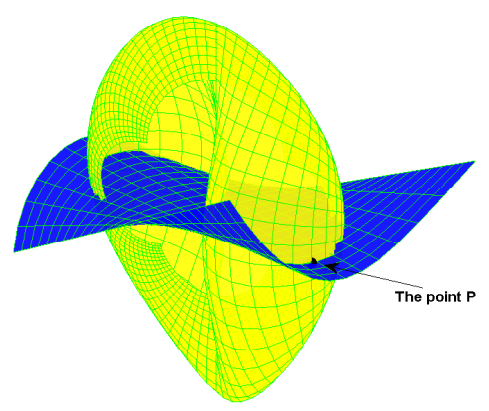

FIGURE 1. Projections of the intersecting surfaces $\mathcal{S}_{1}$ and $\mathcal{S}_{2}$.

It is easy to see that we have a transversal intersection at $P$ since $\left\{X_{u_{1}}, X_{u_{2}}\right.$, $\left.Y_{w_{1}}\right\}$ is linearly independent. Then, since $\left\{X_{u_{1}}, X_{u_{2}}, \mathbf{e}_{1}\right\}$ is linearly independent, by using (4.1) we obtain the basis vectors of the normal space of $\mathcal{S}_{1}$ as

$$
\begin{aligned}
& \mathbf{N}_{1}^{X}=X_{u_{1}} \times X_{u_{2}} \times \mathbf{e}_{1}=-\mathbf{e}_{4}, \\
& \mathbf{N}_{2}^{X}=X_{u_{1}} \times X_{u_{2}} \times \mathbf{N}_{1}^{X}=-\mathbf{e}_{1} .
\end{aligned}
$$

Similarly, since $\left\{Y_{w_{1}}, Y_{w_{2}}, \mathbf{e}_{4}\right\}$ is linearly independent at $P$, by using (4.2), we obtain the basis vectors of the normal space of $\mathcal{S}_{2}$ as

$$
\begin{aligned}
& \mathbf{N}_{1}^{Y}=Y_{w_{1}} \times Y_{w_{2}} \times \mathbf{e}_{4}=\mathbf{e}_{3} \\
& \mathbf{N}_{2}^{Y}=Y_{w_{1}} \times Y_{w_{2}} \times \mathbf{N}_{1}^{Y}=-\mathbf{e}_{4} .
\end{aligned}
$$

Hence, $\left\{\mathbf{N}_{1}^{X}, \mathbf{N}_{2}^{X}, \mathbf{N}_{1}^{Y}\right\}$ is linearly independent, i.e. $d=3$. Thus, by using (4.3) we find the tangent vector of the intersection curve at $P$ as

$$
\mathbf{V}_{1}=\frac{\mathbf{N}_{1}^{X} \times \mathbf{N}_{2}^{X} \times \mathbf{N}_{1}^{Y}}{\left\|\mathbf{N}_{1}^{X} \times \mathbf{N}_{2}^{X} \times \mathbf{N}_{1}^{Y}\right\|}=(0,-1,0,0)
$$

which yields $u_{1}^{\prime}=-1, u_{2}^{\prime}=0, w_{1}^{\prime}=0, w_{2}^{\prime}=-1$.

For the second order derivatives of the given surfaces at $P$ we have

$$
\begin{array}{ll}
X_{u_{1} u_{1}}=(-3,-1,0,0), & Y_{w_{1} w_{1}}=(0,0,0,0), \\
X_{u_{1} u_{2}}=(0,0,-1,1), & Y_{w_{1} w_{2}}=(0,0,0,0), \\
X_{u_{2} u_{2}}=(-1,1,0,0), & Y_{w_{2} w_{2}}=(0,0,2,0) .
\end{array}
$$

Since we may write

$$
\alpha^{\prime \prime}=\lambda_{1} \mathbf{N}_{1}^{X}+\lambda_{2} \mathbf{N}_{2}^{X}+\lambda_{3} \mathbf{N}_{1}^{Y}
$$


by using (4.5) and (4.6) we obtain $\lambda_{1}=0, \lambda_{2}=3, \lambda_{3}=2$. Thus, we have $\alpha^{\prime \prime}=(-3,0,2,0)$ and $u_{1}^{\prime \prime}=1, u_{2}^{\prime \prime}=2, w_{1}^{\prime \prime}=-3, w_{2}^{\prime \prime}=0$ at $P$. Also, we obtain the second Frenet vector of the intersection curve as $\mathbf{V}_{2}=$ $\left(-\frac{3}{\sqrt{13}}, 0, \frac{2}{\sqrt{13}}, 0\right)$, and the first curvature as $k_{1}=\sqrt{13}$ at $P$.

For the third order derivatives of the given surfaces at $P$ we have

$$
\begin{array}{ll}
X_{u_{1} u_{1} u_{1}}=(9,-9,0,0), & Y_{w_{1} w_{1} w_{1}}=(0,0,0,0), \\
X_{u_{1} u_{2} u_{1}}=(0,0,-1,-3), & Y_{w_{1} w_{2} w_{1}}=(0,0,0,0), \\
X_{u_{1} u_{2} u_{2}}=(-1,-3,0,0), & Y_{w_{1} w_{2} w_{2}}=(0,0,2,0), \\
X_{u_{2} u_{2} u_{2}}=(0,0,-3,3), & Y_{w_{2} w_{2} w_{2}}=(0,0,0,6) .
\end{array}
$$

If we use (4.8), we may write

$$
\alpha^{\prime \prime \prime}=-k_{1}^{2} \mathbf{V}_{1}+\mu_{1} \mathbf{N}_{1}^{X}+\mu_{2} \mathbf{N}_{2}^{X}+\mu_{3} \mathbf{N}_{1}^{Y},
$$

where $\mu_{1}=6, \mu_{2}=\mu_{3}=0$. Thus, we have $\alpha^{\prime \prime \prime}=(0,13,0,-6)$ and $u_{1}^{\prime \prime \prime}=1$, $u_{2}^{\prime \prime \prime}=-6, w_{1}^{\prime \prime \prime}=0, w_{2}^{\prime \prime \prime}=13$ at $P$. The third Frenet vector is found as $\mathbf{V}_{3}=(0,0,0,-1)$, and the second curvature is obtained as $k_{2}=\frac{6}{\sqrt{13}}$ at $P$.

If we continue in a similar manner, we find

$$
\mathbf{V}_{4}=\left(-\frac{2}{\sqrt{13}}, 0,-\frac{3}{\sqrt{13}}, 0\right), \quad k_{3}=\frac{47}{\sqrt{13}}
$$

5.2. Example in $\mathbb{E}^{5}$. Let $\mathcal{S}_{1}$ and $\mathcal{S}_{2}$ be the surfaces given by

$$
\begin{aligned}
& X\left(u_{1}, u_{2}\right)=\left(u_{2}^{2}, u_{2}, u_{1}^{3}, u_{1}, u_{1}^{4}\right), \\
& Y\left(w_{1}, w_{2}\right)=\left(w_{1}, w_{2}, w_{2}^{3}, w_{2}, w_{1}^{2}\right),
\end{aligned}
$$

respectively. Let us find the Frenet vectors and curvatures of their intersection curve $\alpha$ at the intersection point $P=X(0,0)=Y(0,0)=(0,0,0,0,0)$. The tangent vector fields of these surfaces are

$$
\begin{aligned}
& X_{u_{1}}=\left(0,0,3 u_{1}^{2}, 1,4 u_{1}^{3}\right), \quad X_{u_{2}}=\left(2 u_{2}, 1,0,0,0\right), \\
& Y_{w_{1}}=\left(1,0,0,0,2 w_{1}\right), \quad Y_{w_{2}}=\left(0,1,3 w_{2}^{2}, 1,0\right) .
\end{aligned}
$$

Then, since $\left\{X_{u_{1}}, X_{u_{2}}, \mathbf{e}_{1}, \mathbf{e}_{3}\right\}$ is linearly independent at $P$, by using (4.1) we obtain the basis vectors of the normal space of $\mathcal{S}_{1}$ as

$$
\begin{aligned}
& \mathbf{N}_{1}^{X}=X_{u_{1}} \times X_{u_{2}} \times \mathbf{e}_{1} \times \mathbf{e}_{3}=\mathbf{e}_{5}, \\
& \mathbf{N}_{2}^{X}=X_{u_{1}} \times X_{u_{2}} \times \mathbf{N}_{1}^{X} \times \mathbf{e}_{1}=\mathbf{e}_{3}, \\
& \mathbf{N}_{3}^{X}=X_{u_{1}} \times X_{u_{2}} \times \mathbf{N}_{1}^{X} \times \mathbf{N}_{2}^{X}=-\mathbf{e}_{1} .
\end{aligned}
$$

Similarly, since $\left\{Y_{w_{1}}, Y_{w_{2}}, \mathbf{e}_{2}, \mathbf{e}_{3}\right\}$ is linearly independent at $P$, by using (4.2) we obtain the basis vectors of the normal space of $\mathcal{S}_{2}$ as

$$
\mathbf{N}_{1}^{Y}=Y_{w_{1}} \times Y_{w_{2}} \times \mathbf{e}_{2} \times \mathbf{e}_{3}=\mathbf{e}_{5}
$$




$$
\begin{aligned}
& \mathbf{N}_{2}^{Y}=Y_{w_{1}} \times Y_{w_{2}} \times \mathbf{N}_{1}^{Y} \times \mathbf{e}_{2}=\mathbf{e}_{3}, \\
& \mathbf{N}_{3}^{Y}=Y_{w_{1}} \times Y_{w_{2}} \times \mathbf{N}_{1}^{Y} \times \mathbf{N}_{2}^{Y}=-\mathbf{e}_{2}+\mathbf{e}_{4} .
\end{aligned}
$$

In this case, $\left\{\mathbf{N}_{1}^{X}, \mathbf{N}_{2}^{X}, \mathbf{N}_{3}^{X}, \mathbf{N}_{3}^{Y}\right\}$ is linearly independent, i.e. $d=4$ at $P$. Thus, by using (4.3) we find the tangent vector of the intersection curve at $P$ as

$$
\mathbf{V}_{1}=\frac{\mathbf{N}_{1}^{X} \times \mathbf{N}_{2}^{X} \times \mathbf{N}_{3}^{X} \times \mathbf{N}_{3}^{Y}}{\left\|\mathbf{N}_{1}^{X} \times \mathbf{N}_{2}^{X} \times \mathbf{N}_{3}^{X} \times \mathbf{N}_{3}^{Y}\right\|}=\left(0, \frac{1}{\sqrt{2}}, 0, \frac{1}{\sqrt{2}}, 0\right)
$$

which yields $u_{1}^{\prime}=\frac{1}{\sqrt{2}}, u_{2}^{\prime}=\frac{1}{\sqrt{2}}, w_{1}^{\prime}=0, w_{2}^{\prime}=\frac{1}{\sqrt{2}}$.

For the nonzero second order derivatives of the given surfaces at $P$ we have $X_{u_{2} u_{2}}=2 \mathbf{e}_{1}, Y_{w_{1} w_{1}}=2 \mathbf{e}_{5}$. Since we may write

$$
\alpha^{\prime \prime}=\lambda_{1} \mathbf{N}_{1}^{X}+\lambda_{2} \mathbf{N}_{2}^{X}+\lambda_{3} \mathbf{N}_{3}^{X}+\lambda_{4} \mathbf{N}_{3}^{Y},
$$

by using (4.5) and (4.6) we obtain $\lambda_{1}=\lambda_{2}=\lambda_{4}=0, \lambda_{3}=1$. Thus, we have $\alpha^{\prime \prime}=\mathbf{N}_{3}^{X}=\mathbf{e}_{1}$ and $u_{1}^{\prime \prime}=u_{2}^{\prime \prime}=w_{2}^{\prime \prime}=0, w_{1}^{\prime \prime}=1$ at $P$. Also, we obtain the second Frenet vector as $\mathbf{V}_{2}=(1,0,0,0,0)$, and the first curvature as $k_{1}=1$ at $P$.

For the nonzero third order derivatives of the given surfaces at $P$ we have $X_{u_{1} u_{1} u_{1}}=6 \mathbf{e}_{3}, Y_{w_{2} w_{2} w_{2}}=6 \mathbf{e}_{3}$. If we use (4.8), we may write

$$
\alpha^{\prime \prime \prime}=-\mathbf{V}_{1}+\mu_{1} \mathbf{N}_{1}^{X}+\mu_{2} \mathbf{N}_{2}^{X}+\mu_{3} \mathbf{N}_{3}^{X}+\mu_{4} \mathbf{N}_{3}^{Y},
$$

where $\mu_{1}=\mu_{3}=\mu_{4}=0, \mu_{2}=-\frac{3}{\sqrt{2}}$. Thus, we have

$$
\alpha^{\prime \prime \prime}=\left(0,-\frac{1}{\sqrt{2}}, \frac{3}{\sqrt{2}},-\frac{1}{\sqrt{2}}, 0\right) \quad \text { and } \quad u_{1}^{\prime \prime \prime}=u_{2}^{\prime \prime \prime}=w_{2}^{\prime \prime \prime}=-\frac{1}{\sqrt{2}}, w_{1}^{\prime \prime \prime}=0
$$

at $P$. The third Frenet vector is found as $\mathbf{V}_{3}=(0,0,1,0,0)$, and the second curvature is obtained as $k_{2}=\frac{3 \sqrt{2}}{2}$ at $P$.

If we continue in a similar manner, we find

$$
\mathbf{V}_{4}=(0,0,0,0,1), \quad \mathbf{V}_{5}=\left(0,-\frac{1}{\sqrt{2}}, 0, \frac{1}{\sqrt{2}}, 0\right), \quad k_{3}=2 \sqrt{2}, \quad k_{4}=0
$$

\section{Conclusion}

Differential geometric properties of the intersection curve of two parametric surfaces are studied in Euclidean $n$-space. We first present the $m$ th order derivative formula of a curve lying on a parametric surface, and then, we obtain curvatures and Frenet vectors of the transversal intersection curve of two parametric surfaces in Euclidean $n$-space. We also provide a computer code produced in MATLAB to simplify determining the coefficients according to the Frenet frame of higher order derivatives of a curve. 


\section{Appendix A}

Let us find the fifth order derivative of $\alpha$ by using (3.1). In this case, since $m=5$, we may write

$$
\begin{aligned}
\alpha^{(5)} & =\mathcal{C}_{1} \sum_{i_{1}=1}^{2} \frac{1}{k_{i_{1}}} X_{i_{1}} u_{i_{1}}^{(5)}+\sum_{r_{1}=1}^{2}\left(\mathcal{C}_{2} \sum_{i_{1}, i_{2}=1}^{2} \frac{1}{k_{i_{1} i_{2} r_{1}}} X_{i_{1} i_{2}} u_{i_{1}}^{\left(5-r_{1}\right)} u_{i_{2}}^{\left(r_{1}\right)}\right) \\
& +\sum_{r_{1}, r_{2}=1}^{2}\left(\mathcal{C}_{3} \sum_{i_{1}, i_{2}, i_{3}=1}^{2} \frac{1}{k_{i_{1} i_{2} i_{1} i_{3} r_{2}}} X_{i_{1} i_{2} i_{3}} u_{i_{1}}^{\left(5-r_{1}-1\right)} u_{i_{2}}^{\left(r_{2}\right)} u_{i_{3}}^{\left(r_{1}-r_{2}+1\right)}\right) \\
& +\sum_{r_{1}, r_{2}, r_{3}=1}^{2}\left(\mathcal{C}_{4} \sum_{i_{1}, i_{2}, i_{3}, i_{4}=1}^{2} \frac{1}{k_{i_{1} i_{2} r_{1} i_{3} r_{2} i_{4} r_{3}}} X_{i_{1} i_{2} i_{3} i_{4}} u_{i_{1}}^{\left(5-r_{1}-2\right)} u_{i_{2}}^{\left(r_{2}\right)} \times\right. \\
& \left.\times u_{i_{3}}^{\left(r_{3}\right)} u_{i_{4}}^{\left(r_{1}-r_{2}-r_{3}+2\right)}\right) \\
& +\mathcal{C}_{5} \sum_{i_{1}, i_{2}, i_{3}, i_{4}, i_{5}=1}^{2} \frac{1}{5 !} X_{i_{1} i_{2} i_{3} i_{4} i_{5}} u_{i_{1}}^{\prime} u_{i_{2}}^{\prime} u_{i_{3}}^{\prime} u_{i_{4}}^{\prime} u_{i_{5} .}^{\prime} .
\end{aligned}
$$

If we consider the definitions of $\mathcal{C}_{i}, 1 \leq i \leq 5$, we get

$$
\begin{aligned}
& \alpha^{(5)}=\left(\begin{array}{l}
5 \\
5
\end{array}\right) \sum_{i_{1}=1}^{2} \frac{1}{k_{i_{1}}} X_{i_{1}} u_{i_{1}}^{(5)}+\left(\begin{array}{l}
5 \\
4
\end{array}\right) \sum_{i_{1}, i_{2}=1}^{2} \frac{1}{k_{i_{1} i_{2}}} X_{i_{1} i_{2}} u_{i_{1}}^{(4)} u_{i_{2}}^{\prime} \\
& +\left(\begin{array}{l}
5 \\
3
\end{array}\right) \sum_{i_{1}, i_{2}=1}^{2} \frac{1}{k_{i_{1} i_{22}}} X_{i_{1} i_{2}} u_{i_{1}}^{\prime \prime \prime} u_{i_{2}}^{\prime \prime} \\
& +\left(\begin{array}{l}
5 \\
3
\end{array}\right)\left(\begin{array}{l}
2 \\
1
\end{array}\right) \sum_{i_{1}, i_{2}, i_{3}=1}^{2} \frac{1}{k_{i_{1} i_{2} i_{31}}} X_{i_{1} i_{2} i_{3}} u_{i_{1}}^{\prime \prime \prime} u_{i_{2}}^{\prime} u_{i_{3}}^{\prime} \\
& +\left(\begin{array}{l}
5 \\
3
\end{array}\right)\left(\begin{array}{l}
2 \\
2
\end{array}\right) \sum_{i_{1}, i_{2}, i_{3}=1}^{2} \frac{1}{k_{i_{1} i_{2} i_{32}}} X_{i_{1} i_{2} i_{3}} u_{i_{1}}^{\prime \prime \prime} u_{i_{2}}^{\prime \prime} u_{i_{3}}^{(0)} \\
& +\left(\begin{array}{l}
5 \\
2
\end{array}\right)\left(\begin{array}{l}
3 \\
1
\end{array}\right) \sum_{i_{1}, i_{2}, i_{3}=1}^{2} \frac{1}{k_{i_{1} i_{2} i_{31}}} X_{i_{1} i_{2} i_{3}} u_{i_{1}}^{\prime \prime} u_{i_{2}}^{\prime} u_{i_{3}}^{\prime \prime} \\
& +\left(\begin{array}{l}
5 \\
2
\end{array}\right)\left(\begin{array}{l}
3 \\
2
\end{array}\right) \sum_{i_{1}, i_{2}, i_{3}=1}^{2} \frac{1}{k_{i_{1} i_{2} i_{32}}} X_{i_{1} i_{2} i_{3}} u_{i_{1}}^{\prime \prime} u_{i_{2}}^{\prime \prime} u_{i_{3}}^{\prime} \\
& +\sum_{r_{1}, r_{2}, r_{3}=1}^{2}\left(\mathcal{C}_{4} \sum_{i_{1}, i_{2}, i_{3}, i_{4}=1}^{2} \frac{1}{k_{i_{1} i_{2 r_{1}} i_{3 r_{2}} i_{4 r_{3}}}} X_{i_{1} i_{2} i_{3} i_{4}} u_{i_{1}}^{\left(5-r_{1}-2\right)} u_{i_{2}}^{\left(r_{2}\right)} \times\right. \\
& \left.\times u_{i_{3}}^{\left(r_{3}\right)} u_{i_{4}}^{\left(r_{1}-r_{2}-r_{3}+2\right)}\right) \\
& +\left(\begin{array}{l}
5 \\
1
\end{array}\right)\left(\begin{array}{l}
4 \\
1
\end{array}\right)\left(\begin{array}{l}
3 \\
1
\end{array}\right)\left(\begin{array}{l}
2 \\
1
\end{array}\right) \sum_{i_{1}, i_{2}, i_{3}, i_{4}, i_{5}=1}^{2} \frac{1}{5 !} X_{i_{1} i_{2} i_{3} i_{4} i_{5}} u_{i_{1}}^{\prime} u_{i_{2}}^{\prime} u_{i_{3}}^{\prime} u_{i_{4}}^{\prime} u_{i_{5}}^{\prime} .
\end{aligned}
$$


However, since the orders of derivatives in the sums

$$
\sum_{i_{1}, i_{2}, i_{3}=1}^{2} \frac{1}{k_{i_{1} i_{1} i_{32}}} X_{i_{1} i_{2} i_{3}} u_{i_{1}}^{\prime \prime \prime} u_{i_{2}}^{\prime \prime} u_{i_{3}}^{(0)} \text { and } \sum_{i_{1}, i_{2}, i_{3}=1}^{2} \frac{1}{k_{i_{1} i_{2} i_{31}}} X_{i_{1} i_{2} i_{3}} u_{i_{1}}^{\prime \prime} u_{i_{2}}^{\prime} u_{i_{3}}^{\prime \prime}
$$

do not satisfy the conditions in Theorem 1, these sum terms must be canceled, i.e. we have

$$
\begin{aligned}
\alpha^{(5)} & =\sum_{i_{1}=1}^{2} \frac{1}{k_{i_{1}}} X_{i_{1}} u_{i_{1}}^{(5)}+5 \sum_{i_{1}, i_{2}=1}^{2} \frac{1}{k_{i_{1} i_{2}}} X_{i_{1} i_{2}} u_{i_{1}}^{(4)} u_{i_{2}}^{\prime} \\
& +10 \sum_{i_{1}, i_{2}=1}^{2} \frac{1}{k_{i_{1} i_{22}}} X_{i_{1} i_{2}} u_{i_{1}}^{\prime \prime \prime} u_{i_{2}}^{\prime \prime}+20 \sum_{i_{1}, i_{2}, i_{3}=1}^{2} \frac{1}{k_{i_{1} i_{2} i_{3}}} X_{i_{1} i_{2} i_{3}} u_{i_{1}}^{\prime \prime \prime} u_{i_{2}}^{\prime} u_{i_{3}}^{\prime} \\
& +30 \sum_{i_{1}, i_{2}, i_{3}=1}^{2} \frac{1}{k_{i_{1} i_{2} i_{32}}} X_{i_{1} i_{2} i_{3}} u_{i_{1}}^{\prime \prime} u_{i_{2}}^{\prime \prime} u_{i_{3}}^{\prime} \\
& +\underbrace{\sum_{i_{1}}^{2}\left(\mathcal{C}_{4} \sum_{i_{1}, i_{2}, i_{3}, i_{4}=1}^{2} \frac{1}{k_{i_{1} i_{2} i_{1}} i_{3 r_{2}} i_{4} r_{3}} X_{i_{1} i_{2} i_{3} i_{4}} u_{i_{1}}^{\left(5-r_{1}-2\right)} u_{i_{2}}^{\left(r_{2}\right)} \times\right.}_{\sum_{1}, r_{2}, r_{3}=1} u_{i_{4}}^{\left(r_{1}-r_{2}-r_{3}+2\right)}) \\
+ & 120 \sum_{i_{1}, i_{2}, i_{3}, i_{4}, i_{5}=1}^{2} \frac{1}{5 !} X_{i_{1} i_{2} i_{3} i_{4} i_{5}} u_{i_{1}}^{\prime} u_{i_{2}}^{\prime} u_{i_{3}}^{\prime} u_{i_{4}}^{\prime} u_{i_{5}}^{\prime} .
\end{aligned}
$$

Similarly, the orders of the derivatives in the underlined sum $\mathbf{I}$ are given below:

\begin{tabular}{|c|c|c|c||c|c|c|c||}
\hline \multicolumn{1}{|c|}{} & \multicolumn{1}{|c|}{} & \multicolumn{1}{|c||}{} & \multicolumn{4}{c||}{ Orders in the sum I } \\
\cline { 5 - 9 } & & & & $t_{1}$ & $t_{2}$ & $t_{3}$ & $t_{4}$ \\
\hline Cases & $r_{1}$ & $r_{2}$ & $r_{3}$ & $5-r_{1}-2$ & $r_{2}$ & $r_{3}$ & $r_{1}-r_{2}-r_{3}+2$ \\
\hline i & 1 & 1 & 1 & 2 & 1 & 1 & 1 \\
\hline ii & 1 & 1 & 2 & 2 & 1 & 2 & 0 \\
\hline iii & 1 & 2 & 1 & 2 & 2 & 1 & 0 \\
\hline iv & 1 & 2 & 2 & 2 & 2 & 2 & -1 \\
\hline v & 2 & 1 & 1 & 1 & 1 & 1 & 2 \\
\hline vi & 2 & 1 & 2 & 1 & 1 & 2 & 1 \\
\hline vii & 2 & 2 & 1 & 1 & 2 & 1 & 1 \\
\hline viii & 2 & 2 & 2 & 1 & 2 & 2 & 0 \\
\hline
\end{tabular}

It is clear here that only the orders in Case i satisfy the condition of Theorem 
1. Therefore, for the sum $\mathbf{I}$ we only have

$$
\left(\begin{array}{l}
5 \\
2
\end{array}\right)\left(\begin{array}{l}
3 \\
1
\end{array}\right)\left(\begin{array}{l}
2 \\
1
\end{array}\right)\left(\begin{array}{l}
1 \\
1
\end{array}\right) \sum_{i_{1}, i_{2}, i_{3}, i_{4}=1}^{2} \frac{1}{k_{i_{1} i_{2 r_{1}} i_{3 r_{2}} i_{4 r_{3}}}} X_{i_{1} i_{2} i_{3} i_{4}} u_{i_{1}}^{\prime \prime} u_{i_{2}}^{\prime} u_{i_{3}}^{\prime} u_{i_{4}}^{\prime} .
$$

If we substitute (A.4) into (A.3), since $k_{i_{1}}=1$ !, $k_{i_{1} i_{21}}=1$ !, $k_{i_{1} i_{22}}=1$ !, $k_{i_{1} i_{2} i_{31}}=2$ !, $k_{i_{1} i_{2} i_{32}}=2$ ! and $k_{i_{1} i_{21} i_{31} i_{41}}=3$ ! according to Table 1 , we obtain

$$
\begin{aligned}
\alpha^{(5)} & =\sum_{i_{1}=1}^{2} X_{i_{1}} u_{i_{1}}^{(5)}+5 \sum_{i_{1}, i_{2}=1}^{2} X_{i_{1} i_{2}} u_{i_{1}}^{(4)} u_{i_{2}}^{\prime}+10 \sum_{i_{1}, i_{2}=1}^{2} X_{i_{1} i_{2}} u_{i_{1}}^{\prime \prime \prime} u_{i_{2}}^{\prime \prime} \\
& +10 \sum_{i_{1}, i_{2}, i_{3}=1}^{2} X_{i_{1} i_{2} i_{3}} u_{i_{1}}^{\prime \prime \prime} u_{i_{2}}^{\prime} u_{i_{3}}^{\prime}+15 \sum_{i_{1}, i_{2}, i_{3}=1}^{2} X_{i_{1} i_{2} i_{3}} u_{i_{1}}^{\prime \prime} u_{i_{2}}^{\prime \prime} u_{i_{3}}^{\prime} \\
& +10 \sum_{i_{1}, i_{2}, i_{3}, i_{4}=1}^{2} X_{i_{1} i_{2} i_{3} i_{4}} u_{i_{1}}^{\prime \prime} u_{i_{2}}^{\prime} u_{i_{3}}^{\prime} u_{i_{4}}^{\prime} \\
& +\sum_{i_{1}, i_{2}, i_{3}, i_{4}, i_{5}=1}^{2} X_{i_{1} i_{2} i_{3} i_{4} i_{5}} u_{i_{1}}^{\prime} u_{i_{2}}^{\prime} u_{i_{3}}^{\prime} u_{i_{4}}^{\prime} u_{i_{5}}^{\prime}
\end{aligned}
$$

\section{Appendix B}

$\mathrm{n}=$ input('enter the size of the space: $\mathrm{n}=$ ');

syms $\mathrm{s}$

$\mathrm{T} 1\{:\}=\operatorname{sprintfc}\left(' k \% \mathrm{~d}(\mathrm{~s})^{\prime}, 1: \mathrm{n}-1\right)$;

$\operatorname{syms}(\mathrm{T} 1\{:\})$

$\mathrm{A} 1=[\mathrm{T} 1\{:\}]$;

$\mathrm{B} 1=\operatorname{str} 2 \operatorname{sym}(\mathrm{A} 1) ;$

$\mathrm{T} 2\{:\}=\operatorname{sprintfc}\left(' \mathrm{~V} \% \mathrm{~d}(\mathrm{~s})^{\prime}, 1: \mathrm{n}\right) ;$

$\operatorname{syms}(\mathrm{T} 2\{:\})$

$\mathrm{A} 2=[\mathrm{T} 2\{:\}]$;

$\mathrm{B} 2=\operatorname{str} 2 \operatorname{sym}(\mathrm{A} 2)$;

$\mathrm{C}(1)=\mathrm{k} 1(\mathrm{~s}) * \mathrm{~V} 2(\mathrm{~s})$;

for $\mathrm{i}=2: \mathrm{n}-1$

$\mathrm{C}(\mathrm{i})=-\mathrm{B} 1(\mathrm{i}-1) * \mathrm{~B} 2(\mathrm{i}-1)+\mathrm{B} 1(\mathrm{i}) * \mathrm{~B} 2(\mathrm{i}+1)$;

end

$\mathrm{C}(\mathrm{n})=-\mathrm{B} 1(\mathrm{n}-1) * \mathrm{~B} 2(\mathrm{n}-1)$;

$\mathrm{D}=\operatorname{diff}(\mathrm{B} 2, \mathrm{~s})$;

$\mathrm{E}=\mathrm{k} 1(\mathrm{~s}) * \mathrm{~V} 2(\mathrm{~s})$;

$\mathrm{F}(1)=\mathrm{V} 1(\mathrm{~s})$;

$\mathrm{F}(2)=\mathrm{k} 1(\mathrm{~s}) * \mathrm{~V} 2(\mathrm{~s})$;

alpha $(1)=\mathrm{V} 1(\mathrm{~s})$;

$\operatorname{alpha}(2)=\mathrm{k} 1(\mathrm{~s}) * \mathrm{~V} 2(\mathrm{~s})$;

$\mathrm{F}(3)=\operatorname{diff}(\mathrm{E}, \mathrm{s})$;

$\operatorname{alpha}(3)=\operatorname{subs}(\mathrm{F}(3), \mathrm{D}(2), \mathrm{C}(2))$; 


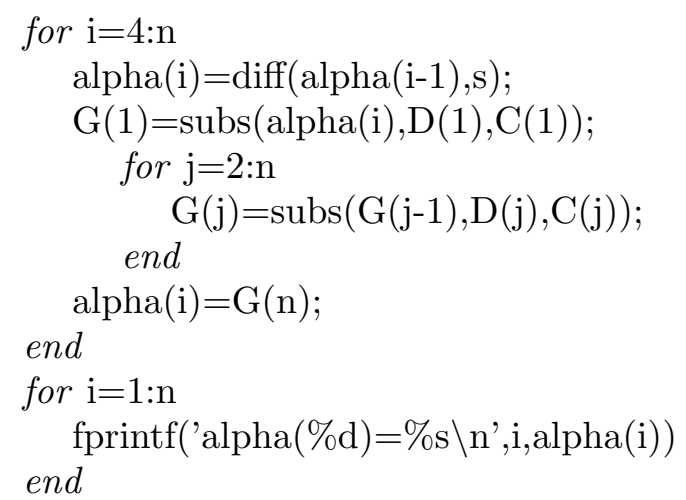

\section{Acknowledgements}

The second author would like to thank TÜBİTAK-BIDDEB for their financial supports during her doctoral studies. The authors thank the anonymous referee for the valuable comments and suggestions which have improved the quality of the paper.

\section{Conflict of interest}

The authors declare that they have no conflict of interest.

\section{References}

[1] N. H. Abdel-All, S. A. Badr, M. A. Soliman, and S. A. Hassan, Intersection curves of hypersurfaces in $\mathbb{R}^{4}$, Comput. Aided Geom. Design 29(2) (2012), 99-108.

[2] N. H. Abdel-All, S. A. Badr, M. A. Soliman, and S. A. Hassan, Intersection curves of two implicit surfaces in $\mathbb{R}^{3}$, J. Math. Comput. Sci. 2(2) (2012), 152-171.

[3] H. S. Abdel-Aziz, M. K. Saad, and A. A. Abdel-Salam, On implicit surfaces and their intersection curve in Euclidean 4-space, Houston J. Math. 40(2) (2014), 339-352.

[4] O. Aléssio, Differential geometry of intersection curves in $\mathbb{R}^{4}$ of three implicit surfaces, Comput. Aided Geom. Design 26(4) (2009), 455-471.

[5] O. Aléssio, Formulas for second curvature, third curvature, normal curvature, first geodesic curvature and first geodesic torsion of implicit curve in n-dimensions, Comput. Aided Geom. Design 29(4) (2012), 189-201.

[6] O. Aléssio, M. Düldül, B. Uyar Düldül, S.A. Badr, and N.H. Abdel-All, Differential geometry of non-transversal intersection of three parametric hypersurfaces in Euclidean 4-space, Comput. Aided Geom. Design 31 (2014), 712-727.

[7] O. Aléssio, M. Düldül, B. Uyar Düldül, S.A. Badr, and N.H. Abdel-All, Differential geometry of non-transversal intersection curves of three implicit hypersurfaces in Euclidean 4-space, J. Comput. Appl. Math. 308 (2016), 20-38.

[8] S. A. Badr, N. H. Abdel-All, O. Aléssio, M. Düldül, and B. Uyar Düldül, Nontransversal intersection curves of hypersurfaces in Euclidean 4-space, J. Comput. Appl. Math. 288 (2015), 81-98.

[9] M. Düldül, On the intersection curve of three parametric hypersurfaces, Comput. Aided Geom. Design 27(1) (2010), 118-127. 
[10] M. Düldül and Ö. Akbaba, Willmore-like methods for the intersection of parametric (hyper)surfaces, Appl. Math. Comput. 226 (2014), 516-527.

[11] H. Gluck, Higher curvatures of curves in Euclidean space, Amer. Math. Monthly 73(7) (1966), 699-704.

[12] R. Goldman, Curvature formulas for implicit curves and surfaces, Comput. Aided Geom. Design 22 (2005), 632-658.

[13] C. M. Hoffmann and J. Zhou, Visualization of surfaces in four-dimensional space, Department of Computer Science Technical Reports, Paper 814, 1990. https://docs.lib.purdue.edu/cstech/814.

[14] S. Hur, T-w. Kim, and C. Bracco, Comprehensive study of intersection curves in $\mathbb{R}^{4}$ based on the system of ODEs, J. Comput. Appl. Math. 256 (2014), 121-130.

[15] M. S. Lone, O. Aléssio, M. Jamali, and M. H. Shahid, Differential geometry of transversal intersection curves of hypersurfaces in $\mathbb{R}^{5}$, J. Dyn. Syst. Geom. Theor. 15(2) (2017), 147-162.

[16] L. S. Moreira, Geometric analogy and products of vectors in $n$ dimensions, Advances in Linear Algebra \& Matrix Theory 3(1) (2013), 1-6.

[17] B. M. Özçetin and M. Düldül, Curvature computations for the intersection curves of hypersurfaces in Euclidean n-space, Comput. Aided Geom. Design 84 (2021), Paper No. 101954, 14 pp.

[18] M. A. Soliman, N. H. Abdel-All, S. A. Hassan, and S. A. Badr, Intersection curves of implicit and parametric surfaces in $\mathbb{R}^{3}$, Appl. Math. 2 (2011), 1019-1026.

[19] B. Uyar Düldül and M. Düldül, The extension of Willmore's method into 4-space, Math. Commun. 17(2) (2012), 423-431.

[20] T. J. Willmore, An Introduction to Differential Geometry, Clarendon Press, Oxford, 1959.

[21] X. Ye and T. Maekawa, Differential geometry of intersection curves of two surfaces, Comput. Aided Geom. Design 16 (1999), 767-788.

Department of Mathematics, Science and Arts Faculty, Yildiz Technical

UNIVERsity, IstanBul, TURKEY

E-mail address: merihoz@yildiz.edu.tr

E-mail address: mduldul@yildiz.edu.tr 